J. Pröll

A. Bensussan

F. Goffin

J.-M. Foidart

A. Berrebi
P. Le Bouteiller

\section{Tubal versus uterine placentation: similar HLA-G expressing extravillous cytotrophoblast invasion but different maternal leukocyte recruitment}

\section{Key words:}

Fallopian tubes; HLA-G; HLA-G monoclonal antibodies; immunohistochemistry; NK cell receptors; placenta

\section{Acknowledgments:}

This work was supported by Institut National de la Santé et de la Recherche Médicale, Association pour la Recherche sur le Cancer (grant 9489), Etablissement Français des Greffes, Université Paul Sabatier and by a grant from the Austrian Science Foundation (J1800MED) to J.P. The gifts of the following mAbs are gratefully acknowledged: CD56 (Dr. A. Moretta, Genova, Italy), CD94 (Dr. M. Lopez-Botet, Madrid), HCA2 and HC10 (Pr. H. Ploegh, Boston, MA), L31 (Dr. A. Beretta, Paris), LIR1/ILT2 (Dr. D. Cosman, Immunex, Seattle, WA), LIR2/ILT4 (Dr. M. Colonna, Basel, Switzerland), 87G (Dr. D. Geraghty, Seattle, WA). We thank Pr. G. Delsol for helpful comments on the manuscript, Dr. P. Cochard for his expertise in confocal microscopy, M. Aguerre-Girr for her excellent technical assistance. Dr. P. Seldmayr is gratefully acknowledged for his participation in part of this work.
Abstract: The nonclassical HLA-G class I gene is expressed by extravillous cytotrophoblast that invades decidua in uterine pregnancy, suggesting that it may contribute to the immunological mechanisms that protect the fetus against maternal alloimmune response and/or pathogen infections. We first addressed the question of whether HLA-G expression was dependent on maternal tissue environment by comparing uterine and ectopic tubal pregnancies. Using HLA-G-specific mAb on placental cryosections, we found by immunohistochemistry that all subtypes of extravillous cytotrophoblast similarly expressed HLA-G in pregnant tubes, demonstrating that its expression was independent of the site of implantation. We next compared by immunohistochemistry the phenotype of maternal leukocytes recruited in both pregnant tissues. In contrast to decidua, pregnant tubes were characterized firstly, by the lack of natural killer (NK) cells and of cells expressing CD94 receptor specific for HLA-E, secondly, by a prominent increase of CD8 + T cells, dendritic cells, and macrophages, the latter co-expressing the LIR1/ILT2 killer immunoglobulin-like receptor (KIR), and finally, by the presence of cells expressing LIR2/ILT4 KIR or BY55 NK receptors, known to bind to HLA-G. Such cell types may favor a unique innate defense in pregnant tubes. These observations also suggest that trophoblast HLA-G expression does not influence the recruitment of particular maternal leukocytes in pregnant tissues.

During pregnancy, trophoblast cells expressing paternal alloantigens invade maternal uterine tissue to form the placenta $(1,2)$. They are faced to the following dilemna: firstly, they should escape a likely maternal anti-paternal alloimmune response, possibly directed by decidual cytotoxic $\mathrm{CD} 8+\mathrm{T}$ and/or natural killer (NK) cells, secondly, in case of viral uterine infection, extravillous cytotrophoblast cells (Evct), that constitute one of the first layers of trophoblast which could be infected, should be recognized and eliminated either by maternal NK cells or self-HLA class I-restricted, anti-viral

\author{
Authors' affiliations: \\ J. Pröll, \\ A. Bensussan ${ }^{2}$, \\ F. Goffin ${ }^{3}$, \\ J.-M. Foidart ${ }^{3}$, \\ A. Berrebi ${ }^{4}$, \\ P. Le Bouteiller ${ }^{1}$ \\ ${ }^{1}$ Institut National de la \\ Santé et de la Recherche \\ Médicale U395, Hôpital de \\ Purpan, Toulouse, France, \\ ${ }^{2}$ INSERM U448,Faculté de \\ Médecine de Créteil, \\ France, \\ ${ }^{3}$ Service de Gynécologie \\ Obstétrique, Hôpital la \\ Citadelle, Liège, Belgium, \\ ${ }^{4}$ Service de Gynécologie \\ Obstétrique, Hôpital La \\ Grave, Toulouse, France \\ Correspondence to: \\ Philippe Le Bouteiller \\ INSERM U395 \\ CHU Purpan \\ BP3028 \\ 31024 Toulouse Cedex 3 \\ France \\ Tel: + 33562748374 \\ Fax: +33 562748386 \\ e-mail: \\ phil..b@@purpan.inserm.fr
}


Fig. 1. Schematic diagram comparing a first trimester pregnancy in human uterus and in Fallopian tube. A) Uterine placentation is characterized by interstitial implantation and decidualization of endometrium, attachment of chorionic villi to the maternal decidua basalis and establisment of a functional blood flow. In tubal implantation, the overall structure of placentation is the same as in uterus, but the ectopic placental bed structure is different (see below). Fallopian tube lies in a peritoneal fold along the superior margin of the broad ligament, the mesosalpinx. It can be divided into intrauterine part, isthmus, ampulla and infundibulum. B) and C) enlarged diagrams corresponding to areas enclosed in boxes shown in A): transverse sections illustrating the major uterine and Fallopian tube implantation sites. B) Intrauterine pregnancy. Anchoring and floating villi bath in maternal blood of the intervillous space. They are constituted of a central mesenchyme separated from the maternal blood by a double layer of inner villous cytotrophoblast and outer syncytiotrophoblast. At the base of cell columns which fix the anchoring villi to the decidua, villous cytotrophoblast becomes proliferative and then invades the decidua basalis (interstitial trophoblast), and breach maternal

peptide $\mathrm{T}$ cells, in order to protect the fetus against an harmful viral spreading $(3,4)$. Among the different mechanisms the conceptus has developed to fulfill these dual functions, one can think that the particular pattern of HLA class I expression exhibited by Evct may be of importance. In contrast to most other somatic tissues, these invasive trophoblast cells do not constitutively express polymorphic HLA-A and HLA-B MHC class Ia surface proteins (5), thus protecting them from attack by maternal T-cell-mediated alloreaction. Conversely, they express the non-polymorphic HLA-G $(5,6)$, and HLAE (7) class Ib and, to a lower level, HLA-C (8).

Several functional properties of HLA-G have been recently demonstrated (reviewed in 9). HLA-G was shown to protect cells from lysis by maternal NK cells in a functional assay (reviewed in 10). Protection of trophoblast cells from NK cell attack is crucial for the outcome of pregnancy since many maternal NK cells have been identified in the decidua at the implantation site (reviewed in 3). HLA-G1, which is the only isoform stably expressed at the cell surface (11), is indeed recognized by different Ig-like receptors expressed by subpopulations of T cells, NK cells, B cells, myelomonocytic cells or dendritic cells, namely LIR1/ILT2, LIR2/ILT4, KIR2DL4 and BY55 (reviewed in 6). HLA-G presents nonamer peptides to HLA-G-restricted T cells $(12,13)$. Therefore, capability of HLA-G to exert viral peptide presentation function is very likely. Finally, the soluble form HLA-G1 was recently shown to trigger Fas/Fas ligand-mediated apoptosis in activated CD8 + cells by interacting with $\mathrm{CD} 8$ (14).

In order to evaluate the in vivo importance of HLA-G in the outcome of pregnancy, we asked the following questions: first, is trophoblast HLA-G expression induced by maternal environment? Second, do HLA-G expressing trophoblast cells participate to the uterine spiral arteries (endovascular trophoblast). These extravillous cytotrophoblast (Evct) cells are therefore in contact with maternal leukocytes, whereas chorionic villus syncytiotrophoblast is in contact with maternal blood leukocytes. C) Ectopic tubal pregnancy. Floating and anchoring chorionic villi exhibit a similar structure with the same mesenchymal differentiation, and normal syncytiotrophoblast and villous cytotrophoblast layers. Anchoring villi similarly attach to the connective tissue components, showing a slightly more prominent development of trophoblastic shell. Invading Evct derived from proliferating trophoblast at the tips of the cell column infiltrate and extensively colonize muscular wall as well as maternal vessels of tubal tissue. Spiral arteries, important for uterine placentation, are absent in Fallopian tube. However, it is well vascularized, maternal vessels providing blood supply into the intervillous space. Therefore, Evct invasion of the tubal wall follows a very similar pattern in both tubal and uterine pregnancies, as already described (21). The wall of the Fallopian tube consists of different layers including muscularis, Lamina propria or connective tissue and serosa. 1, 2, 3 and 4 inserts correspond to the sites of sections shown in Figs. $2-5$.

recruitment of defined maternal leukocytes, NK cells in particular, at the sites of implantation? Such questions have been addressed in the present study by comparing HLA-G expression in normal uterine and in ectopic tubal pregnancies (Fig. 1) and by examining the phenotype of maternal leukocytes recruited in both pregnant tissues. By immunohistochemical analysis, we found that Evct, both in uterine and in tubal pregnancies, similarly express HLA-G. This suggests that HLA-G expression is endogenously regulated through transcription factors and therefore depends on the state of differentiation but not on the maternal environment. When pregnancies develop outside the natural uterine tissue, in Fallopian tubes, our immunohistochemical study revealed a different leukocyte phenotype profile, including a lack of NK cells and a presence of CD8+ cells, dendritic cells and cells expressing different Ig-like receptors recognizing HLA-G. This further suggests that HLA-G does not participate to the recruitment of maternal leukocytes to the sites of implantation.

\section{Material and methods}

\section{Tissues}

Seven surgically removed Fallopian tubes containing ectopic pregnancies were examined. The clinically estimated ages of the tubal pregnancies ranged from 8 to 10 weeks postconception. No sign of salpingitis or infection such as cellular infiltrates into stroma and lumen was detectable by histopathology evaluation. Normal nonpregnant control Fallopian tubes were either obtained from two non menopaused women undergoing hysterectomy due to benign cause 


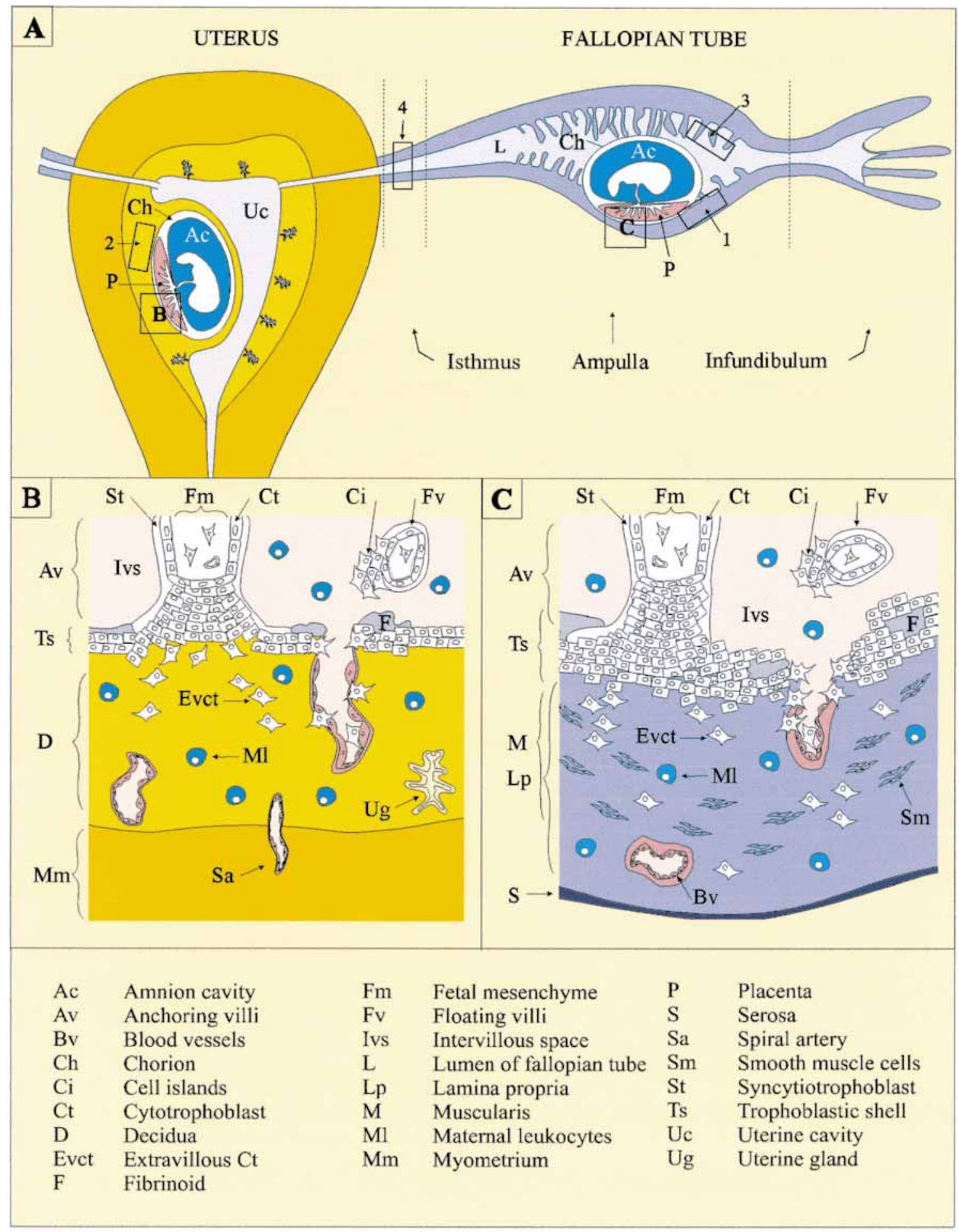

or from a woman having evolutive intrauterine pregnancy of 12 weeks: at the time of elective abortion, a tubal sterilisation was performed by partial salpingectomy. Fragments of chorionic villi and samples from decidua were obtained from three women under- going elective terminations of 10 to 12 weeks normal pregnancies with no known abnormality. Tissues were obtained from patients at La Grave hospital, Toulouse, France and la Citadelle hospital, Liège, Belgium. Tissues were dissected to include implantation sites 
of either tubal or uterine pregnancies, as well as tubal wall of isthmic and ampullar regions (tubal pregnancies) and decidua basalis (uterine pregnancies). They were placed into O.C.T. embedding compound (Miles Incorporation, Elkhart, IN, USA) and immediately snap frozen and stored in liquid nitrogen until cryostat sectioning. All procedures and consent forms were approved by the local ethical commitees.

\section{Antibodies}

Characteristics and source of the mAbs used in this study are listed in Table 1.

\section{Immunohistochemistry}

Serial cryostat sections $(5 \mu \mathrm{m})$ from the tissues described above were air-dried onto frosted slides (Erie Scientific Company, Portsmouth $\mathrm{NH}, \mathrm{USA}$ ) for $2 \mathrm{~h}$, fixed in acetone at room temperature for $5 \mathrm{~min}$ and rehydrated with phosphate-buffered saline (PBS) followed by an incubation for $15 \mathrm{~min}$ with $\mathrm{Ab}$ diluent (Dako, Glostrup, Denmark) containing 10\% normal human serum. Sections were then incubated with primary $\mathrm{mAb}$ at appropriate dilution for 30 min. After washing with $\mathrm{PBS} / 0.01 \%$ Tween 20 (Sigma-Aldrich Chemie Gmbh, Steinheim, Germany) the slides were incubated with biotinylated goat anti-mouse IgG secondary Ab (LSAB 2 kit; Dako) for $10 \mathrm{~min}$ and rinsed with PBS/Tween 20. Slides were then incu-
Primary monoclonal antibodies used in the study*

\begin{tabular}{|c|c|c|c|}
\hline Clone & Isotype & Ligand & Source \\
\hline OV-TL 12/30 & IgG1 & Cytokeratin 7 & Dako, Glostrup, Denmark \\
\hline 3B4 & IgG2a & Vimentin & Dako \\
\hline $87 \mathrm{G}$ & IgG2a & HLA-G & gift of Dr. D. Geraghty, Seattle, WA, USA \\
\hline MEM-G/1 & $\operatorname{lgG1}$ & HLA-G & Exbio, Praha, Czech Republic \\
\hline W6/32 & $\lg G 2 a$ & HLA-G,-A,-B,-C,-E & Coulter-Immunotech \\
\hline HCA2 & IgG1 & HLA-G,-A,-E (free hc) & gift of Pr. H. Ploegh, Boston, MA, USA \\
\hline HC10 & $\operatorname{lgG} 2 a$ & HLA-B,-C (free hc) & gift of Pr. H. Ploegh \\
\hline L31 & IgG1 & HLA-C (free hc) & gift of Dr. A. Beretta, Paris, France \\
\hline 010 & IgG1 & CD1a & INSERM U448, Paris** \\
\hline $4 \mathrm{~A} 7.6$ & $\lg G 2 a$ & $\mathrm{CD} 1 \mathrm{~b}$ & INSERM U448, Paris** \\
\hline L161 & $\operatorname{lgG1}$ & CD1c & INSERM U448, Paris** \\
\hline 0516 & $\operatorname{lgG} 2 a$ & CD4 & INSERM U448, Paris** \\
\hline $\operatorname{CD} 8 \times 8$ & $\lg G 2 b$ & CD8 & INSERM U448, Paris** \\
\hline BE48 & IgG2a & CD8 & INSERM U448, Paris** \\
\hline GPE3 & $\operatorname{lgG1}$ & CD14 & INSERM U448, Paris** \\
\hline CR16 & IgG1 & CD16 & INSERM U448, Paris** \\
\hline CR20 & $\operatorname{lgG1}$ & CD20 & INSERM U448, Paris** \\
\hline M-A251 & IgG1 & CD25 & Pharmingen, San Diego, CA, USA \\
\hline C-218 & IgG1 & CD56 & gift of Dr. A. Moretta, Genova, Italy \\
\hline HB-15A & $\lg G 2 b$ & CD83 & Coulter-Immunotech \\
\hline HP-3B1 & $\operatorname{lgG} 2 a$ & CD94 & gift of Dr. M. Lopez-Botet, Madrid, Spain \\
\hline BMA 031 & $\lg G 2 b$ & pan TCR $\alpha / \beta$ & Coulter-Immunotech \\
\hline IMMU 389 & $\operatorname{lgG1}$ & TCR V $\delta 2$ & Coulter-Immunotech \\
\hline B8.12.2 & $\lg G 2 b$ & HLA-DR & Coulter-Immunotech \\
\hline M407 & $\operatorname{lgG1}$ & LIR1/ILT.2 & gift of Dr. D. Cosman, Immunex, Seattle, WA, USA \\
\hline 42D1 & Rat IgG1 & LIR2/ILT4 & gift of Dr. M. Colonna, Basel, Switzerland \\
\hline TM-60 & IgG1 & BY55 & INSERM U448, Paris** \\
\hline
\end{tabular}

Table 1 
Fig. 2. Expression of HLA-G by extravillous cytotrophoblast in a tubal pregnancy: immunohistochemical staining. Cryosections were incubated with primary $\mathrm{mAb}$, biotinylated goat anti-mouse IgG $\mathrm{Ab}$, streptavidin-peroxidase and $\mathrm{AEC}+$ substrate and counterstained with hemalum. A) placental section in the ampullary region (see insert 1 of Fig. $1 \mathrm{~A}$ and its enlargement in Fig. 1C): staining with 87G HLA-G $\mathbf{m A b}$. Cell islands (Ci) of floating villi are strongly stained, whereas both villous cytotrophoblast (Ct) and syncytiotrophoblast (St) as well as fetal mesenchyme (Fm) of chorionic villi are negative. B) Serial section stained with cytokeratin $7 \mathrm{mAb}$, a trophoblast-specific marker. Extravillous cytotrophoblast (Evct) in cell island (Ci) is stained, demonstrating the trophoblast origin of HLA-G expressing cells. Both villous cytotrophoblast and syncytiotrophoblast are also stained, but not fetal mesenchyme (Fm). C) Another section showing a tubal placental bed stained with $87 \mathrm{G}$ HLA-G mAb. Evct HLA-G-positive cells are prominent in the trophoblastic shell (Ts) and show invasion (arrowheads) in muscularis (M) and lamina propria $(\mathrm{Lp})$, near the serosa $(\mathrm{S}) .(\times 125)$.

bated with peroxidase blocking solution (Dako) for $15 \mathrm{~min}$ at room temperature and incubated with streptavidin-peroxidase (LSAB 2 kit; Dako) for $10 \mathrm{~min}$. The peroxidase staining was developed with 3-amino-9-ethylcarbazole (AEC+, Dako). After extensive washes in distilled water, slides were counterstained with Mayer's hemalum solution (Merk, Darmstadt, Germany) and mounted in Faramount (Dako). Negative control slides were incubated with a mixture of mouse IgG1, IgG2a and IgG2b isotypic control mAbs (Dako). Photographs were taken with a Zeiss Axioskope microscope (Zeiss, Oberkochen, Germany).

\section{Double-labeling immunofluorescence}

Tissues were treated as described above. For double immunostaining, sections were first incubated with Ab diluent blocking solution (Dako) containing 10\% normal human serum for $15 \mathrm{~min}$ at room temperature, then with primary $\mathrm{Ab}$ at $4^{\circ} \mathrm{C}$ for $30 \mathrm{~min}$, washed in $\mathrm{PBS} / 0.01 \%$ Tween 20 for $30 \mathrm{~min}$ and incubated with the secondary biotinylated anti-mouse IgG Ab $\left(30 \mathrm{~min}, 4^{\circ} \mathrm{C}\right)$. After washing in PBS $\left(30 \mathrm{~min}, 4^{\circ} \mathrm{C}\right.$ ) sections were incubated with rhodamine-conjugated streptavidine (3.4 $\mu \mathrm{g} / \mathrm{mL}$; Coulter-Immunotech, Marseille, France). Following another washing step, slides were incubated with FITCconjugated $\mathrm{mAb}$ for $30 \mathrm{~min}$ at $4^{\circ} \mathrm{C}$. After extensive washing in PBS for $2 \mathrm{~h}$, sections were mounted in Moviol containing 2.5\% 1,4-diazobicyclo-octane (DABCO; Sigma) as a fading retardant. Controls were done by incubation with isotypic control mouse Ig (mixture of IgG1, IgG2a and IgG2b, Dako, Trappes, France), followed by biotinylated anti-mouse IgG Ab and rhodamine-conjugated streptavidine or by FITC-conjugated mouse IgG (Coulter Immunotech, Marseille, France). Results were examined using a laser scanning confocal microscope (Zeiss).

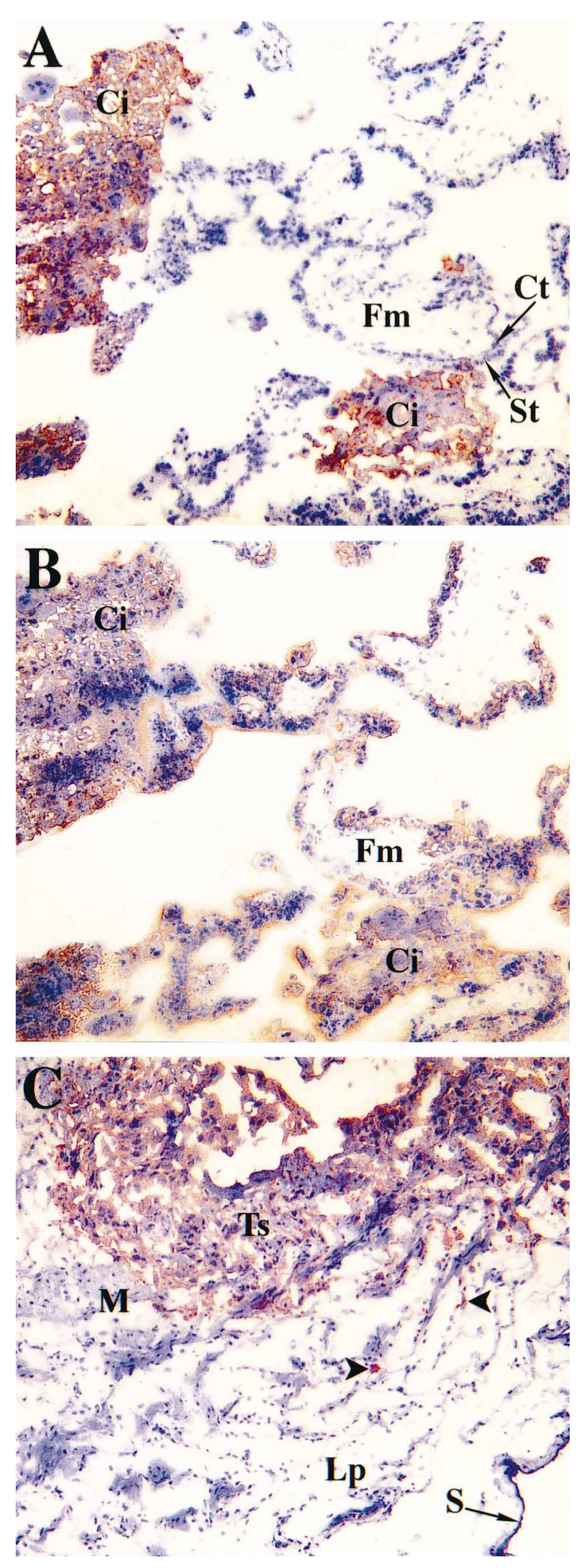

Tissue Antigens 2000: 56: 479-491 


\section{Statistical analysis}

A Mann and Whitney test was used.

\section{Results}

\section{Invasive extravillous cytotrophoblast in tubal pregnancies express HLA-G}

A comparative immunohistochemical study, using different HLA-G mAbs, was performed on frozen sections of implantation sites and chorionic villi taken from pregnant first trimester Fallopian tube and uterus (see Fig. 1). $87 \mathrm{G} \mathrm{mAb}$ strongly stained Evct present in cell islands sprouting from floating villi in Fallopian tube (Fig. 2A). In contrast, villous cytotrophoblast and syncytiotrophoblast of chorionic villi were unreactive. Trophoblast nature of these HLA-G expressing cells was identified by the use of trophoblast specific, cytokeratin $7 \mathrm{mAb}$ (15): in a serial section, this $\mathrm{mAb}$ specifically labeled the cells that were also HLA-G positive (Fig. 2B). By contrast, these cells did not react with vimentin mAb (Table 2). Cytotrophoblastic shell as well as Evct infiltrating the tubal muscularis were also stained with $87 \mathrm{G}$ (Fig. 2C), as were Evct invading maternal blood vessels (data not shown). No trophoblast giant cells could be seen in pregnant tubes. Two other HLA-G mAbs, namely MEM-G/1 and HCA2, gave a similar staining pattern (Table 2). Some mesenchymal cells in the chorionic villi also appeared positive with these latter mAbs. By comparison, W6/32 mAb, which recognizes a framework determinant on all HLA class heavy chains associated with $\beta 2 \mathrm{~m}$ (16), also stained Evct as well as most mesenchy- mal cells, whereas cytotrophoblast and syncytiotrophoblast remain negative (Table 2). It was previously reported that, in addition to HLA-G, Evct invading the decidua also expressed HLA-C (8). HLAG positive Evct cells in pregnant tubes similarly expressed HLA-C, as demonstrated by their staining with $\mathrm{HC} 10$ and L31, HLA-C mAbs (Table 2). Incubations of the same sections with isotypic Ig control Abs always gave negative stainings (data not shown). The same results were reproduced on three different tubal pregnancies.

By comparison, immunohistochemical study performed with the same mAbs on uterine first trimester placenta similarly detected expression of HLA-G and HLA-C in evct (data not shown), confirming previous studies $(3,5,17,18)$. Our report therefore clearly demonstrates that the different Evct subpopulations invading a pregnant Fallopian tube strongly express HLA-G, like in normal uterine pregnancy.

\section{Lack of CD56+, maintenance of CD14+ and CD4+ and increasing number of CD8,$+ C D 1+$ and $\gamma / \delta$ T cells in pregnant Fallopian tubes}

In order to evaluate whether HLA-G expressing cells recruited particular types of maternal cells, we then compared the maternal leukocyte populations present in uterine or tubal pregnancies. We performed an immunohistochemical study on decidua or pregnant tubal cryosection by the means of a series of markers, namely CD56 (NK-cell marker), CD14 (macrophage), $\alpha / \beta$ or $\gamma / \delta$ T-cell receptors, CD4, CD8 (T cell), CD1a, CD1b, CD1c or CD83 (mostly dendritic cell), CD20 (mostly B cell), CD16 (NK cells, monocytes, macrophages, granulocytes), HLA-DR (mostly antigen presenting cells) and CD25 (activated T, B cells, macrophages). Results are illustrated in Fig. 3,

\begin{tabular}{|c|c|c|c|c|c|}
\hline \multirow[b]{2}{*}{$\mathrm{mAb}$} & \multirow[b]{2}{*}{ Specificity } & \multicolumn{3}{|c|}{ Trophoblast cell subpopulations } & \multirow[b]{2}{*}{ Fetal mesenchyme } \\
\hline & & Evct & vct & st & \\
\hline $87 G$ & HLA-G & +++ & - & - & - \\
\hline MEM-G/1 & HLA-G & ++ & - & - & $-/+$ \\
\hline HCA2 & HLA-G,-A,-E (free hc) & ++ & - & - & $-/+$ \\
\hline W6/32 & HLA-G,-A,-B,-C,-E & +++ & - & - & $+/+++$ \\
\hline HC10 & HLA-B,C (free hc) & ++ & - & - & $-/++$ \\
\hline L31 & HLA-C (free hc) & ++ & - & - & $-/++$ \\
\hline OV-TL 12/30 & cytokeratin 7 & +++ & +++ & +++ & - \\
\hline 3B4 & vimentin & - & - & - & ++ \\
\hline
\end{tabular}

Table 2 

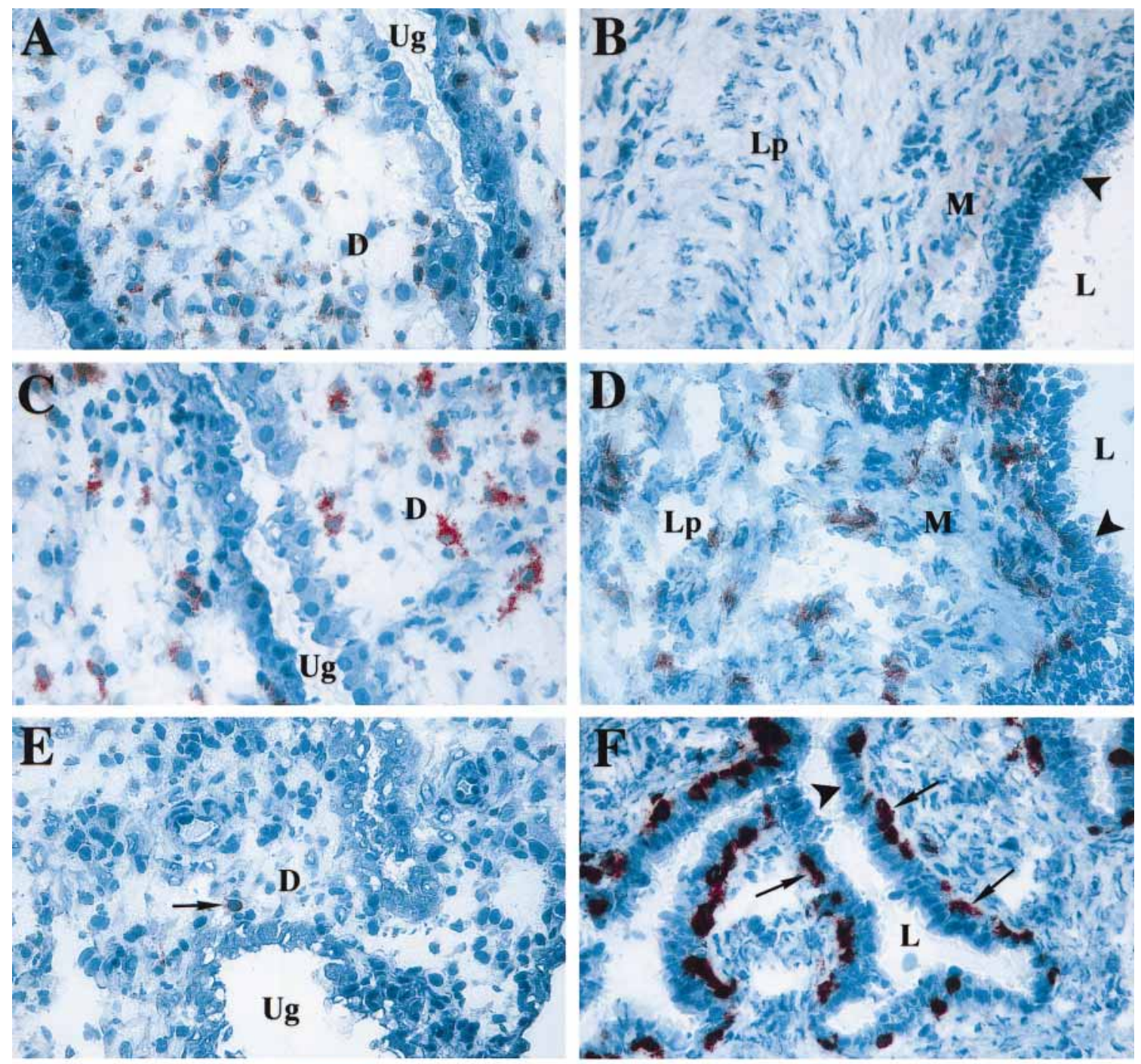

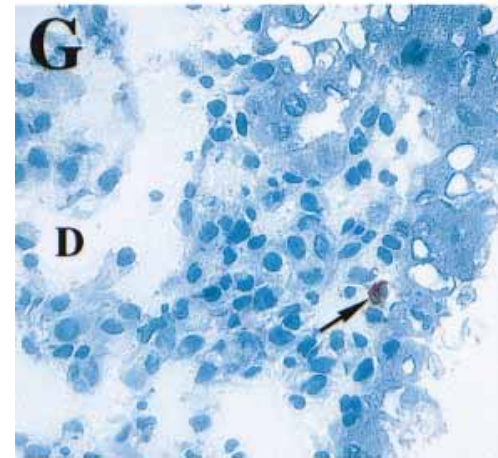

Fig. 3. Expression of CD56 (A and B), CD14 (C and D), CD8 (E and $\mathrm{F}$ ) and $\mathrm{CD} 1 \mathrm{a}(\mathrm{G}$ and $\mathrm{H})$ : comparative immunohistochemical analysis (immunoperoxidase staining) in first trimester decidua basalis (A, C, E, G, insert 2 of Fig. 1A) and pregnant Fallopian tubes (B, D, ampulla, F, H, isthmus, inserts 3 and 4 of Fig. 1A, respectively). CD56 staining reveals many positive leukocytes in the decidua (A) but not in Fallopian tubes (B). Only few cells which exhibit

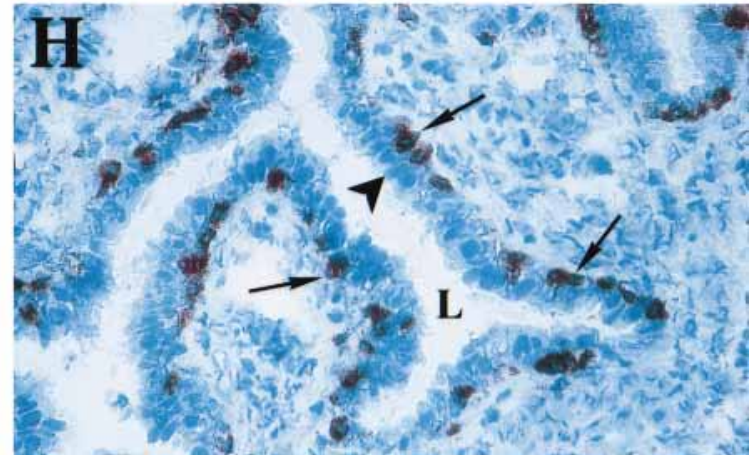

smooth muscle morphology appear very faintly stained. Almost equal CD14 positive cell density is observed in both tissues (C and D). Very few CD8 and CD1a stained cells are detectable in the decidua basalis (E and G, respectively) whereas many of them are present in tubal epithelium of the isthmus (F and $\mathrm{H}$, arrows). D, decidua; $\mathrm{Ug}$, uterine glands; Lp, Lamina propria; L, lumen; M, muscularis; arrow heads: Fallopian tube epithelium. $(\times 228)$. 


\begin{tabular}{|c|c|c|c|c|c|c|}
\hline \multicolumn{4}{|c|}{ Intrauterine pregnancy } & \multicolumn{3}{|c|}{ Fallopian tube pregnancy } \\
\hline $\begin{array}{l}\text { Phenotypic } \\
\text { markers }\end{array}$ & Localization & $\begin{array}{l}\text { Cell } \\
\text { density }\end{array}$ & $\begin{array}{l}\text { Staining } \\
\text { intensity }\end{array}$ & Localization & $\begin{array}{l}\text { Cell } \\
\text { density }\end{array}$ & $\begin{array}{l}\text { Staining } \\
\text { intensity }\end{array}$ \\
\hline CD56 & db-int & $\underline{+++}$ & $\underline{+++}$ & & - & - \\
\hline CD14 & db-int & +++ & +++ & isth, amp - Ip, m & ++ & +++ \\
\hline CD4 & db-int & + & +++ & isth, amp - Ip, m & + & +++ \\
\hline CD8 & $\mathrm{db}$-int & + & ++ & isth, amp - Ip, m, ep & $\underline{++}$ & $\underline{+++}$ \\
\hline CD1a & db-ep & $-/+$ & +++ & isth, amp - Ip, m, ep & $\underline{+++}$ & +++ \\
\hline $\mathrm{CD} 1 \mathrm{~b}$ & & - & - & isth, amp - Ip, m, ep & ++ & +++ \\
\hline CD1c & db-ep & $-/+$ & +++ & isth, amp - Ip, m, ep & $\underline{++}$ & +++ \\
\hline CD83 & & - & - & isth, amp - Ip, m, ep & $\underline{-1+}$ & ++ \\
\hline CD20 & & - & - & & - & - \\
\hline CD25 & & - & - & & - & - \\
\hline CD16 & db-int & + & +++ & isth, amp - Ip, m & + & +++ \\
\hline $\operatorname{TCR} \alpha / \beta$ & db-int & - & - & isth, amp - Ip, m & \pm & $\underline{++}$ \\
\hline TCR V $\delta 2$ & db-int & + & + & isth, amp - Ip, m & + & ++ \\
\hline HLA-DR & db-int & +++ & +++ & isth, amp - Ip, m, ep & +++ & +++ \\
\hline LIR1/ILT2 & db-int & $-/+$ & $-/+$ & isth, amp - Ip, m, ep & $\underline{-1++}$ & $\underline{++}$ \\
\hline LIR2/ILT4 & & - & - & isth, amp - m & $-1+$ & \pm \\
\hline BY55 & & - & - & isth, amp - m & $-1+$ & \pm \\
\hline CD94 & db-int & $\underline{+++}$ & $\underline{+++}$ & & $-/+$ & + \\
\hline \multicolumn{7}{|c|}{$\begin{array}{l}\text { Cell density: }- \text {, negative; }-/+ \text {, very few; }+ \text {, few; }+++ \text {, many; Staining intensity: }- \text {, negative; }+ \text {, faintly positive; }++ \text {, positive; } \\
+++ \text {, strongly positive; Localization: db, decidua basalis; Ip, lamina propria; } m \text {, mucosa; int, interstitial; ep, epithelial; isth, isthmus; } \\
\text { amp, ampulla }\end{array}$} \\
\hline
\end{tabular}

summarized in Table 3 and quantified in Fig. 4. The decidua contains a large number of CD56 + NK cells (Fig. 3A). In contrast, no CD56+ leukocyte was detected in Fallopian tubes (Fig. 3B). The decidua also contains many CD14+ cells (Fig. 3C), whereas less are detected in tubal epithelium (Fig. 3D). A higher number of CD14+ cells was observed in the isthmus as compared with the ampullar region of the tube. There were few CD4 weakly positive cells in decidua and tube (Table 3).

When CD8 and CD1a markers were examined, it appeared that no or very few cells were positively stained in decidual tissue (Fig. $3 \mathrm{E}, \mathrm{G}$, respectively), whereas tubal epithelia did contain many of them (Fig. 3F, H, respectively). CD1a + cells were mainly located at the base of epithelial cells (Fig. 3F). In the isthmus, a high number of CD1a-, CD1b- and CD1c-positive cells was also found (Table 3). Another dendritic marker (CD83) stained only very few cells in the isthmus and the ampulla (Table 3) exhibiting typical dendritic morphology. We observed that the number of CD1a-, CD1b-, CD1c-positive cells was higher in the isthmus than in the ampulla.
Table 3 summarizes expression of other markers: T-cell receptor (TCR) $\alpha / \beta$ expressing cells, absent in decidua, were detected in low numbers in tubes. Some $\gamma \delta$-positive T cells, identified by the V $\delta 2$ staining, were observed in decidua but more were present in pregnant tubes. Immunostaining with CD20 B-cell marker or CD25, interleukin (IL)-2 receptor activation marker was negative in either tissue. A low number of CD16+ cells was found in uterus and Fallopian tube. The number of CD16+ cells was more important in the subepithelial regions of the ampulla than in the isthmus. The number and staining intensity of HLA-DR + cells were almost identical in both uterus and tubes (Table 3). HLA-DR staining was particularly intense in mucosal epithelial cells lining the lumen of Fallopian tubes. By double labeling, we found that some CD1a+ cells also expressed HLA-DR (data not shown). There was no difference for either of the markers analysed above between the tubal implantation site and the tubal mucosa away from the implantation site.

We then analysed whether expression of these different leukocyte markers was similar in non pregnant Fallopian tubes obtained 


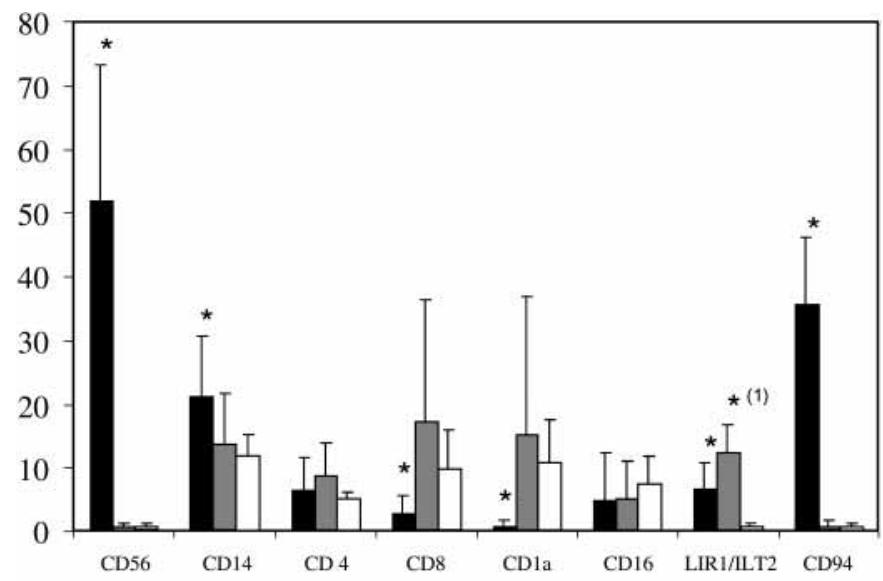

Pregnant uterus $\square$ Pregnant Fallopian tube $\square$ Non-pregnant Fallopian tube

Fig. 4. Comparison between the maternal leukocyte populations in pregnant uterus and pregnant Fallopian tubes (implantation regions) as well as between pregnant and non pregnant Fallopian tubes: statistical analysis. Frozen sections of decidua and pregnant tubes were labelled with CD56, CD14, CD4, CD8, CD1a, CD16, LIR1/ILT2, or CD94, followed by an avidin-biotin immunoperoxidase staining system. Columns represent the mean percentage of positive cells for a given marker obtained from 4,000 cell counts on ten different fields of $218 \mu \mathrm{m}^{2}$ of tissue (mean of three different samples $+/-\mathrm{SD}$ ). The non parametric Mann-Whitney U test was used to make statistical comparison between pregnant uterus (decidua basalis) and Fallopian tube (ampullar/or isthmic regions). (1): Statistical comparison between pregnant and non pregnant Fallopian tube. $* p<0.05$.

from a woman with uncomplicated uterine pregnancy or from a non pregnant woman undergoing hysterectomy: no significant difference was observed between the three kinds of tubes (Fig. 4).

Altogether, these results showing that tubal leukocyte phenotype profile was very different from the one observed in decidua, suggest that HLA-G expressing cells did not recruit particular maternal cell types.

\section{Several HLA-G recognizing Ig-like receptors are up-regulated in Fallopian tubes}

Knowing that HLA-G is the ligand of several Ig-like receptors that are known to modulate cell lysis and also to induce particular cytokine secretion reviewed in (6), we then investigated whether some maternal leukocytes potentially in contact with evct, did express some of these receptors. We performed a comparative immunohistochemical analysis of three of these receptors, namely LIR1/ILT2, LIR2/ILT4 killer Ig-like receptors (KIR) and BY55 NK receptor, in decidua and pregnant tube cryosections, using specific mAbs. Whereas very few and faintly positive LIR1/ILT2 expressing cells were detected in decidua basalis of intrauterine pregnancy (Fig. 5A), a higher number of stained cells was observed in the intra- and subepithelial regions of pregnant Fallopian tube (Fig. 5B). There was no preferential localization at the implantation site, since positively stained cells were also observed in other parts of the tube, mainly in the isthmus and also, but in lower number, in the ampullar epithelial region (Table 3). When pregnant and non-pregnant Fallopian tubes were examined, it appeared that the number of LIR1/ILT2 positive cells was significantly enhanced in pregnant tubes (Fig. 4). Few LIR2/ILT4 and BY55 slightly positive cells were also detected in pregnant tubes but not in decidua (Table 3).

By comparison, we also studied expression of another receptor, CD94, known to associate with NKG2 lectin-like subunits to form an heterodimer and to recognize a nonclassical MHC class I ligand, HLA-E (19, 20). Whereas many CD94 strongly stained cells were observed in the decidua (Fig. $5 \mathrm{C}$ ), very few were detected in pregnant tube (Fig. 5D), showing that this lectin-like receptor was also differentially expressed, according to normal or ectopic sites of implantation. These results are summarized in Table 3 and quantified in Fig. 4.

\section{LIR1/ILT2+ cells co-express CD14}

In order to define the phenotype of LIR1/ILT2 expressing cells found in relatively great number in pregnant tubes, we performed a two-color laser scanning microscopy analysis. Since NK cells and $\mathrm{B}$ cells were almost absent in tubes, and that CD1a expressing cells were mostly concentrated on epithelium whereas LIR1/ILT2-positive cells were scattered in all parts of the tube, suggesting that these two markers were not expressed by the same cells, we used CD14 in combination with LIR1/ILT2 mAbs. Such a dual labeling revealed that LIR1/ILT2 co-localized with CD14 macrophage marker (Fig. 6). All isotypic controls were negative (data not shown).

\section{Discussion}

In the present study, we demonstrated by immunohistochemistry that evct similarly expressed HLA-G in tubal or uterine placenta, but we observed that maternal tubal and decidual leukocytes populations significantly differ in their composition. The following conclusions can be drawn from these informative observations: i) HLA$\mathrm{G}$ is expressed by invasive evct, independently of the maternal tissue environment; ii) HLA-G is unlikely to control the recruitment of maternal leukocytes to the site of implantation; iii) NK cells might not be essential for proper implantation and outcome of pregnancy; iv) finally, tubal leukocyte composition may favor unique innate 

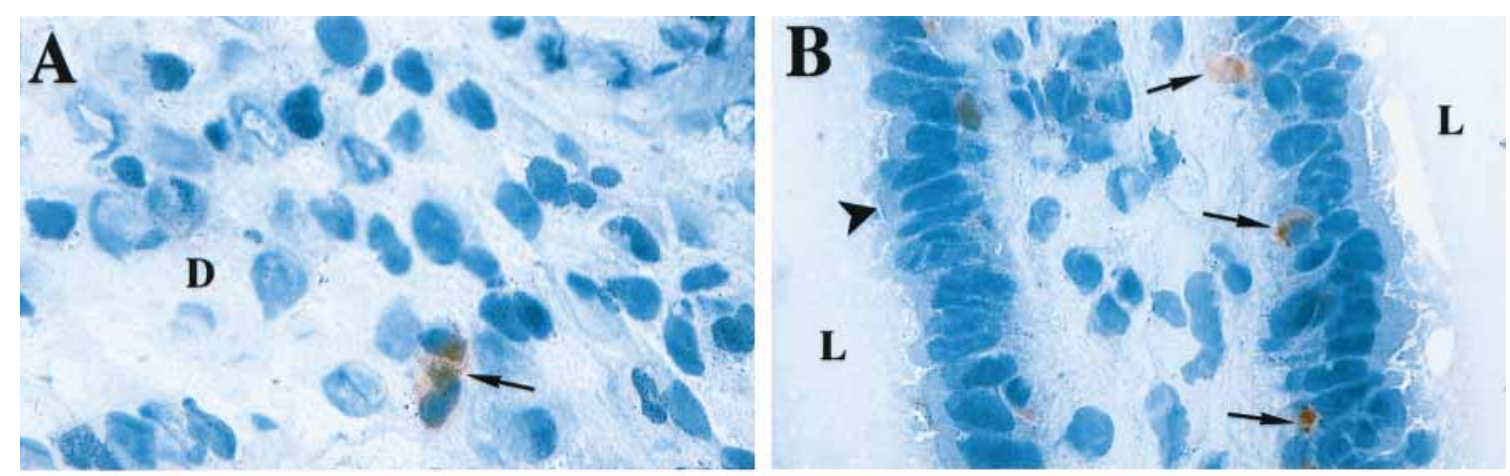

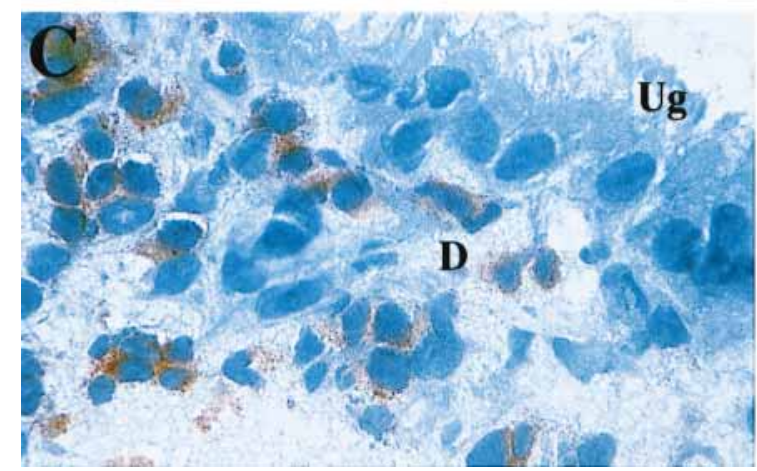

Fig. 5. Comparative immunohistochemical analysis of LIR1/ILT2 (A and B) and CD94 (C and D) expression in first trimester human decidua (A and $C$, insert 2 of Fig. 1A) and pregnant Fallopian tubes (B and D, insert 3 of Fig. 1A): same immunoperoxidase staining system as in Figs. 2 and 3. Very few LIR1/ ILT2 expressing cells

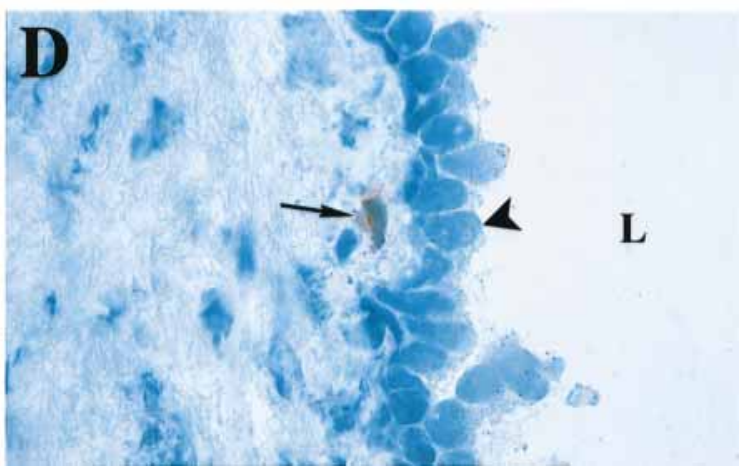

are present in the decidua (A), whereas some intraepithelial cells are stained in tubal epithelium (arrows) (B). In contrast, many CD94 stained cells are present in the decidua basalis $(\mathrm{C})$, whereas very few are detectable in tubal epithelium (D). D, decidua; L, lumen; Ug, uterine gland. Arrow heads: Fallopian tube epithelium. $(\times 570)$. defense and local mucosal immunity without compromising potential pregnancy.

HLA-G is expressed by invasive Evct, independently of the maternal tissue environment. On the contrary, we observed that non invasive trophoblast subpopulations, namely villous cytotrophoblast and syncytiotrophoblast, did not express HLA-G in either tissue. Using different HLA-G mAbs, we have observed that the different subpopulations of evct in pregnant Fallopian tubes were strongly stained and followed a pattern very similar to the one observed in pregnant uterus that is, in trophoblastic cell islands and trophoblastic shell as well as on evct penetrating the muscularis and maternal vessels. These observations suggest that HLA-G expression is not dependent upon the maternal microenvironment which differs in many ways between uterus and tubes, including tissue structure (21), leukocyte populations (22 and this study), cytokine or hormone secretion (23). Indeed HLA-G expression was described at much earlier stages of development, in human pre-implantation blastocysts (24), not yet in contact with maternal tissue. HLA-G may thus favor attachment of blastocyst to the maternal tissue either uterus, Fallopian tube or likely any other potential ectopic sites.
HLA-G is unlikely to control the recruitment of maternal leukocytes to the site of implantation. Different observations made in this study support this hypothesis. Firstly, despite the presence of similar HLA-G expressing Evct in both pregnant tissues, decidual and tubal leukocyte composition significantly differ. In addition to the up-regulation of some Ig-like cell receptors, we observed a shift of leukocyte distribution in favour of CD8, CD1 and $\gamma / \delta$ expressing cells, as well as a lack of CD56+ and CD94+ cells in Fallopian tubes. Secondly, we did not notice a preferential localization of these leukocytes at areas of strong HLA-G-positive trophoblast shell invasion in tubal pregnancies. CD8, CD1, $\gamma / \delta$, as well as LIR1/ILT2-, LIR2/ILT4- and BY55-positive cells, were detected both in ampulla and isthmus, therefore far away from the site of implantation.

NK cells might not be essential for proper implantation and outcome of pregnancy. No evidence was found in our study for the presence of CD56 + cells in pregnant tubes resembling the major decidual leukocyte population reproducibly detected in pregnant uterus $((3,5,25)$ and this study). Such a lack of tubal CD56 expressing cells was also observed in previous studies (22, 25-27). Al- 

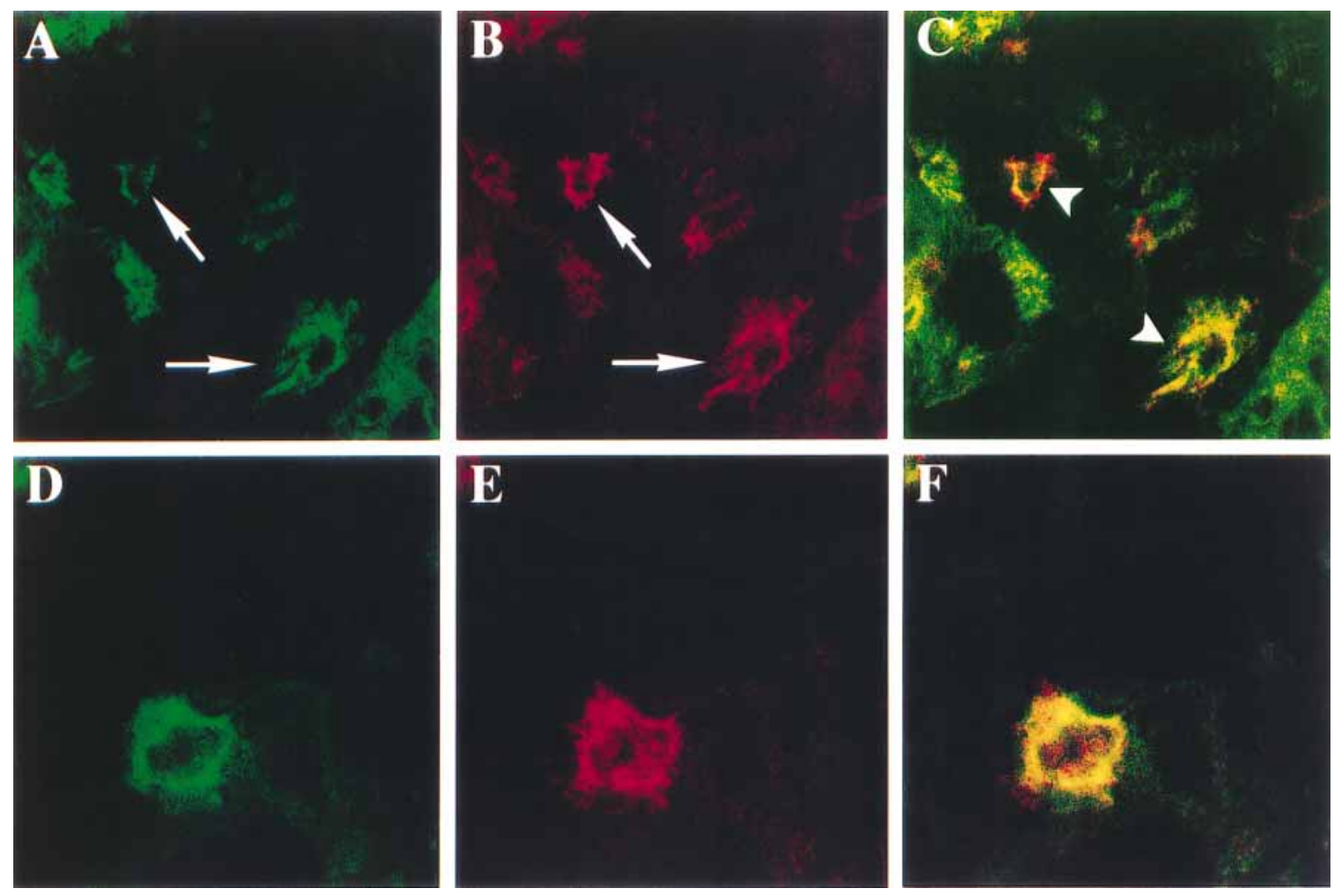

Fig. 6. Colocalization of CD14 and LIR/ILT2 in pregnant Fallopian tubes. Pregnant tubal sections were fixed in acetone and incubated with FITC-labelled CD14 (A and D, green) or with LIR1/ILT2, followed by biotinylated anti-mouse IgG and rhodamine-conjugated streptavidin (B and $\mathrm{E}$, red) and were imaged by confocal laser scanning microscopy. (C and F) An overlay of A and B or D and E, respectively, where yellowish coloring (arrow heads) indicates colocalization of CD14 and LIR1/ILT2. The pictures are representative of three independent experiments. Upper panels $(\times 630)$. Lower panels $(\times 2016)$. though we cannot exclude that few CD56 $6^{\text {dull }}$ cells were detectable in our immunohistochemical study, these observations suggest that NK cells might not be essential for normal implantation and successful outcome of pregnancy. Despite some vascular abnomalities and small placental size which have been detected in pregnant mice depleted of NK cells (28), there is so far no direct proof that the absence of NK cells prevents pregnancy success.

Tubal leukocyte composition may favor unique innate defense and local mucosal immunity without compromising potential pregnancy. Fallopian tubes have been considered as part of the mucosal immune system, due in particular to the expression of IgA receptors (29). Our observations fit with this interpretation. Firstly, we observed that two types of professional antigen presenting cells were particularly abundant in tubes: macrophages and dendritic cells, which are known to participate to the immediate defense against bacteria at vital tissues sites. Macrophages were identified by the CD14 marker (3). In addition to their antigen presenting function, placental macrophages were shown to activaly participate in antiinflammatory process characterized by a Th2-associated effector function with a high capacity of phagocytosis (25). CD14 was re- cently shown to mediate clearance of apoptotic cells without inciting inflammation (30). Functions of $\mathrm{CD} 14+$ tubal macrophages are likely to be the same. Dendritic cell phenotype was deduced by the following markers: CD1a, CD1b, CD1c, CD83 and HLA-DR (31, 32) CD1 expressing cells recruit $\gamma \delta \mathrm{T}$ cells (which were also detected in tubes) as well as NK T cells and present unique microbial lipids and glycolipids and therefore diversifye the antigenic repertoire presented to T cells (33). Cells expressing these different markers were all localized in the same areas, mostly intraepithelial, and were particularly abundant in isthmus at the uterine entrance. Secondly, tubal leukocyte phenotypic pattern resembles the one of intestinal intraepithelial lymphocytes (IEL) which is CD8+, CD1a+, CD56and BY55+ (34). BY55 is a GPI-anchored protein with a single Iglike domain highly homologous to the pregnancy-specific glycoprotein and weakly homologous to KIR, mostly to KIR2DL4 (34). It has been shown that HLA-G is one of the BY55 ligands and that optimal binding required a prior aggregation of MHC class I complexes (35). The triggering of MHC class I molecules on human Tcell clones expressing BY55 was shown to deliver a potent proliferative signal in the presence of soluble CD3 mAb (35). Such effector 
cell proliferation could thus contribute to an efficient immune response even in the absence of CD28 co-stimulation. In addition to BY55, we demonstrated in this study that some tubal IEL also expressed LIR1/ILT2 (36), in greater number in pregnant than in non pregnant tubes and than in decidua, as well as LIR2/ILT4 Ig-like receptors (37), both of them being ligands of HLA-G (38) and expressed in particular on blood monocytes (39). By using double immunofluorescence staining, we found in this study that LIR1/ILT2 receptor was expressed by CD14+ cells in tubes, which contrasted with decidua in which cells expressing LIR1/ILT2 were shown to be confined to CD56+ (40). Such inhibitory receptors are known to regulate NK- and T-cell cytotoxicity as well as to inhibit or down modulate monocyte activation signals (41). In addition, it was recently shown that HLA-G tetramers with self peptide bound to CD14+ blood monocytes expressing LIR2/ILT4 and also LIR1/ILT2 (38). When specific mAb will be available, it would be interesting to examine in situ expression of another Ig-like cell receptor, namely
KIR2DL4, capable of sensing the HLA-G expression, and previously reported to be expressed on isolated decidual NK cells (40).

In conclusion, during uterine pregnancy, the presence in decidua of many NK cells and cells expressing CD94 receptor specific for HLA-E suggest a prominent function of this latter nonclassical HLA class I molecule. HLA-G, through its leader sequence, may positively regulate expression of HLA-E (42). During tubal pregnancy, HLA-G may modulate the myelomonocytic cell behaviour. The presence of HLA-G-specific receptors on this cell type may influence the inflammatory response to pathogens and cytotoxicity as well as cytokine production and therefore participate to the first line of defense against tubal infections. Tubal leukocytes should protect female reproductive tract without compromising fetal survival in case of pregnancy. Constant expression of HLA-G by Evct in both tubal and uterine placentation strongly suggests important functions of this molecule at the feto-maternal interface during the establishment of pregnancy at either site of implantation.

\section{References}

1. Benirschke K, Kaufmann P. Pathology of the human placenta. New York: Springer, 1995: 871.

2. Cross JC, Werb Z, Fisher S. Implantation and the placenta: key pieces of the development puzzle. Science 1994: 266 1508-18.

3. Loke YW, King A. Human implantation: cell biology and immunology. Cambridge: Cambridge University Press, 1995: 299.

4. King A, Loke Y. Maternal-fetal immune interactions: implications for intrauterine infections - A review. Trophoblast Res 1998: 12: $151-60$

5. Hunt JS. HLA and the maternal-fetal relationship. Austin, TX: R. G. Landes Company, 1996: 192.

6. Le Bouteiller P, Solier C, Pröll J, AguerreGirr M, Fournel S, Lenfant F. Placental HLA-G protein expression in vivo: where and what for? Hum Reprod Update 1999: 5: 223-33.

7. King A, Allan D, Bowen M et al. HLA-E is expressed on trophoblast and interacts with CD94/NKG2 receptors on decidual NK cells. Eur J Immunol 2000: 30: 1623-31.

8. King A, Burrows T, Hiby S et al. Surface expression of HLA-C antigen by human extravillous trophoblast. Placenta 2000: 21: 376-87.
9. Le Bouteiller P, Blaschitz A. The functionality of HLA-G is emerging. Immunol Rev 1999: 167: 233-44.

10. Yokoyama W. The mother-child union: the case of missing-self and protection of the fetus. Proc Natl Acad Sci U S A 1997: 94: 5998-6000.

11. Mallet V, Pröll J, Solier C et al. The full length HLA-G1 and no other alternative form of HLA-G is expressed at the cell surface of transfected cells. Hum Immunol 2000: 61: 212-24.

12. Lee N, Malacko AR, Ishitani A et al. The membrane-bound and soluble forms of HLA-G bind identical sets of endogenous peptides but differ with respect to TAP association. Immunity 1995: 3: 591-600.

13. Schmidt CM, Garrett E, Orr HT. Cytotoxic T lymphocyte recognition of HLA-G in mice. Hum Immunol 1997: 55: 127-39.

14. Fournel S, Aguerre-Girr M, Huc X et al. Cutting Edge: Soluble HLA-G1 triggers CD95/CD95 ligand-mediated apoptosis in activated CD8 + cells by interacting with CD8. J Immunol 2000: 164: 6100-4.

15. Haigh T, Chen C, Jones C, Aplin J. Studies of mesenchymal cells from 1st trimester human placenta: expression of cytokeratin outside the trophoblast lineage. Placenta 1999: 20: 615-25.
16. Barnstable CJ, Bodmer WF, Brown G et al. Production of monoclonal antibodies to group A erythrocytes, HLA and other human cell surface antigens-new tools for genetic analysis. Cell 1978: 14: 9-20.

17. McMaster MT, Librach CL, Zhou Y et al. Human placental HLA-G expression is restricted to differentiated cytotrophoblasts. J Immunol 1995: 154: 3771-8.

18. Blaschitz A, Lenfant F, Mallet $\mathrm{V}$ et al. Endothelial cells in chorionic fetal vessels of first trimester placenta express HLA-G. Eur I Immunol 1997: 27: 3380-8.

19. Braud VM, Allan DS, O'Callaghan CA et al HLA-E binds to natural killer cell receptors CD94/NKG2A, B and C. Nature 1998: 391: 795-9.

20. Lee N, Llano M, Carretero M et al. HLA-E is a major ligand for the natural killer inhibitory receptor CD94/NKG2A. Proc Natl Acad Sci U S A 1998: 95: 5199-204.

21. Randall S, Buckley C, Fox H. Placentation in the Fallopian tube. Int J Gynecol Pathol 1987: 6: 132-9

22. Marx L, Arck P, Kapp M, Kieslich C, Dietl J. Leukocyte populations, hormone receptors and apoptosis in eutopic and ectopic first trimester human pregnancies. Hum Reprod 1999: 14: 1111-7. 
23. Carson SA, Buster JE. Ectopic pregnancy. New Engl J Med 1993: 329: 1174-81.

24. Jurisicova A, Casper RF, MacLusky NJ, Mills GB, Librach CL. HLA-G expression during preimplantation human embryo development. Proc Natl Acad Sci U S A 1996: 93: 161-5.

25. Bulmer J. Immune cells in decidua. In: Kurpisz M, Fernandez N, eds. Immunology of reproduction. Oxford: BIOS Scientific Publishers Ltd, 1995: 313-34.

26. Givan A, White H, Stern J et al. Flow cytometric analysis of leukocytes in the human female reproductive tract: comparison of Fallopian tube, uterus, cervix, and vagina. Am J Reprod Immunol 1997: 38: 350-9.

27. Vassiliadou N, Bulmer J. Characterization of tubal and decidual leukocyte populations in ectopic pregnancy: evidence that endometrial granulated lymphocytes are absent from tubal implantation site. Fertil Steril 1998: 69: 760-7.

28. Guimond M, Luross J, Wang B, Terhorst C, Danial S, Croy B. Absence of Natural Killer cells during murine pregnancy is associated with reproductive compromise in TgE26 mice. Biol Reprod 1997: 56: 16979.

29. Wira C, Richardson J, Prabhala R.

Endocrine regulation of mucosal immunity: effect of sex hormones and cytokines on the afferent and efferent aims of the immune system in the female reproductive tract. In: Ogra P, Mestecky J, Lamm M, Strober W, McGhee J, Bienenstock J, eds. Handbook of mucosal immunity. New York: Academic Press, Inc, 1994: 705-18.
30. Gregory C. CD14-dependent clearance of apoptotic cells: relevance to the immune system. Curr Opin Immunol 2000: 12: $27-$ 34.

31. Melian A, Beckman E, Porcelli S, Brenner M. Antigen presentation by CD1 and MHCencoded class I-like molecules. Curr Opin Immunol 1996: 8: 82-8.

32. Steinman R, Pack M, Inaba K. Dendritic cells in the T-cell areas of lymphoid organs. Immunol Rev 1997: 156: 25-37.

33. Porcelli S, Modlin R. The CD1 system: antigen-presenting molecules for T-cell recognition of lipids and glycolipids. Ann Rev Immunol 1999: 17: 297-329.

34. Anumantha A, Bensussan A, Boumsell L et al. Cloning of BY55, a novel Ig superfamily member expressed on NK cells, CTL, and intestinal intraepithelial lymphocytes. J Immunol 1998: 161: 278090.

35. Agrawal S, Marquet J, Freeman GJ et al. Cutting edge: MHC class I triggering by a novel cell surface ligand costimulates proliferation of activated human $\mathrm{T}$ cells. J Immunol 1999: 162: 1223-6.

36. Colonna M, Navarro F, Bellon T et al. A common inhibitory receptor for major histocompatibility complex class I molecules on human lymphoid and myelomonocytic cells. J Exp Med 1997: 186: 1809-18.

37. Colonna M, Samaridis J, Cella M et al. Human myelomonocytic cells express an inhibitory receptor for classical and nonclassical MHC class I molecules. J Immunol 1998: 160: 3096-100.
38. Navarro F, Llano M, Bellon T, Colonna M, Geraghty DE, Lopez-Botet M. The ILT2(LIR1) and CD94/NKG2A NK cell receptors respectively recognize HLA-G1 and HLA-E molecules co-expressed on target cells. Eur J Immunol 1999: 29: 27783.

39. Allan DS, Colonna M, Lanier LL et al. Tetrameric complexes of human histocompatibility leukocyte antigen (HLA)-G bind to peripheral blood myelomonocytic cells. J Exp Med 1999: 189: 1149-56.

40. Ponte M, Cantoni C, Biassoni R et al. Inhibitory receptors sensing HLA-G1 molecules in pregnancy: deciduaassociated natural killer cells express LIR-1 and CD94/NKG2A and acquire p49, an HLA-G1-specific receptor. Proc Natl Acad Sci U S A 1999: 96: 5674-9.

41. Fanger N, Cosman D, Peterson L, Braddy S, Maliszewski C, Borges L. The MHC class I binding proteins LIR-1 and LIR-2 inhibit Fc receptor-mediated signaling in monocytes. Eur J Immunol 1998: 28: 3423-34.

42. Llano M, Lee N, Navarro F et al. HLA-Ebound peptides influence recognition by inhibitory and triggering CD94/NKG2 receptors: preferential response to an HLAG-derived nonamer. Eur J Immunol 1998: 28: $1-10$. 